\title{
Diagnosis and Treatment of Femoral Head Osteonecrosis: A Protocol for Development of Evidence Based Clinical Practice Guidelines
}

Edward Cheng ( $\square$ cheng002@umn.edu)

University of Minnesota https://orcid.org/0000-0001-6125-9671

Michael A. Mont

Northwell Health Orthopaedics, Lenox Hill Hospital

Stuart B. Goodman

Stanford University Medical Center

Rafael J Sierra

Mayo Clinic College of Medicine and Science

Quanjun Cui

University of Virginia

\section{Hytham S Salem}

Northwell Health Orthopaedics, Lenox Hill Hospital

ARCO Clinical Practice Guidelines Group

$\mathrm{N} / \mathrm{A}$

\section{Protocol}

Keywords: Osteonecrosis, Hip, Diagnosis, Treatment, Clinical practice guidelines

Posted Date: October 1st, 2020

DOl: https://doi.org/10.21203/rs.3.rs-77450/v1

License: (c) (i) This work is licensed under a Creative Commons Attribution 4.0 International License. Read Full License 


\section{Abstract}

Background: There are many treatment options for patients who have osteonecrosis of the femoral head (ONFH) and management strategies vary widely both among and within individual countries. Although many researchers have attempted to elucidate the optimal strategies for managing this disease, the lack of large-scale randomized control trials and the lack of agreement on disease staging have curtailed the development of clear-cut guidelines.

Methods: The Association Research Circulation Osseous (ARCO) group sought to address three questions for the management of patients who have ONFH: (1) What imaging studies are most sensitive and specific for the diagnostic evaluation of patients who have ONFH?; (2)What is the best treatment strategy for preventing disease progression in patients who have pre-collapse lesions?; and (3) What is the best treatment strategy for patients who have post-collapse disease? The PICO (Patient, Intervention, Comparison, and Outcome) format was used to formulate the search strategy for each research question. A systematic review will be performed according to the Preferred Reporting Items for Systematic Reviews and Meta-Analyses (PRISMA) guidelines. ARCO participants have been allocated to three groups, each representing one of the PICO questions. After qualitative and quantitative analysis of the data extracted from studies pertaining to each of the three research questions, a set of evidence-based clinical practice guidelines will be proposed for the management of patients who have ONFH.

Discussion: It is not always clear which treatment method is optimal for the management of ONFH. Thus, many surgeons have developed and performed various procedures based on various patient-specific factors. As there is no consensus on the optimal treatment for various stages of disease, it was clear that developing evidence-based clinical practice guidelines would provide more structure and uniformity to management of these patients. Therefore, the results of this systematic review will lead to the development guidelines that may improve patient-care strategies and result in better outcomes for patients who have ONFH.

Systematic Review Registration: Registration with PROSPERO has been submitted and is pending.

\section{Background}

There are many treatment options for patients who have ONFH and management strategies vary widely both among and within individual countries. Although researchers have attempted to elucidate the optimal strategies for managing this disease, the lack of large-scale randomized control trials and the lack of agreement on disease staging have limited the development of clear-cut guidelines. In addition, cultural differences in patient goals and expectations, differences in the opinions of treating surgeons, and differences in the availability and access to various implants and technologies preclude widespread international agreement on optimal treatments. At a given stage of disease, it is not always clear which treatment method is superior to another. Hence, many surgeons have developed and performed various procedures based on factors such as patient age, associated risk factors, extent of femoral head 
involvement, location of the infarct within the femoral head, and degree of collapse [8]. As there is no consensus on the optimal treatment for ONFH, it was clear that developing evidence-based clinical practice guidelines (CPGs) would provide more structure and uniformity, and therefore, hopefully leading to improved patient-care strategies and outcomes.

CPGs are meant to aid clinicians and patients in determining the most appropriate diagnostic and therapeutic strategies [12]. Our CPG will follow best practice as defined by GRADE ( https://www.gradeworkinggroup.org/) to satisfy the AGREE II checklist criteria [13]. Some key elements include: (1) a panel of experts from multiple disciplines; (2) a PRISMA compliant (attachment 1) systematic review of the current literature;[14] (3) consideration of important patient subgroups and patient preferences; (4) clear and transparent methodology to minimize bias and conflicts of interest; (5) description of the relationship between various treatment modalities and clinical outcomes; and (6) grading the quality of evidence used to guide each recommendation $[12,15-18]$.

In September 2019, the ARCO board voted to pursue the development of a CPG. Shortly thereafter, an individual with expertise in CPG methodology (C.B.) was retained to facilitate a scientifically sound process. Next, the research questions were proposed and circulated to the ARCO board for approval using a process that will be described in the following section. Registration with PROSPERO has been submitted and is pending.

The ARCO board utilized the standardized PICO (Patient, Intervention, Comparison, and Outcome) format for stating the research questions that would drive the clinical practice guideline development. The PICO formula facilitates the literature search strategy used to screen for and identify the evidence needed to answer the proposed research question. In December 2019, the ARCO board convened to develop three research questions that were then proposed and circulated among the ARCO board members for approval. These research questions are were designed to focus upon clinical questions that all physicians must contemplate when managing patients. The ARCO board approved the following PICO questions:

PICO \#1: In patients undergoing diagnostic evaluation for ONFH, what imaging studies are most sensitive and specific for:
a) Diagnosis?
b) Detecting subchondral fracture?
c) Monitoring the effect of any intervention?
d) Correlating symptomatic versus asymptomatic disease?

PICO \#2: In patients who have ARCO stage 1-2 ONFH (without femoral head subchondral fracture or collapse): 
a) What treatment is best at preventing femoral head subchondral fracture?

b) For asymptomatic patients, does treatment versus serial observation reduce the risk of femoral head subchondral fracture?

c) For symptomatic patients, should total hip arthroplasty (THA) be performed to reduce pain?

PICO \#3: In patients who have ARCO stage 3 ONFH (with a femoral head fracture, with or without collapse) what surgical treatment (e.g., rotational osteotomy, hemiarthroplasty, surface replacement arthroplasty, total hip arthroplasty) yields the best functional outcome for patients with:

a) lifespan less than 25 years? (Older patients)

b) lifespan greater than 25 years? (Younger patients)

In summary, this paper will describe the process and development of the guidelines for (1) the diagnosis of ONFH; (2) the treatment of pre-collapse ONFH; and (3) the treatment of post-collapse ONFH.

\section{Methods/design}

Study Type

For PICO \#1, we will aim to include studies that focus on diagnostic imaging, not on a treatment outcome. For PICO \#2 and \#3, prospective and retrospective level of evidence I to IV studies of nontraumatic ONFH will be included. Case reports and review papers will be excluded for PICO \#1, \#2, and \#3.

\section{Eligibility Criteria}

Animal studies, articles without a full text English translation, and studies in which the mean patient age is less than 16 years will be excluded from the search results of PICO \#1, \#2, and \#3.

In order to meet eligibility criteria for PICO \#2 and \#3, studies must use advanced imaging (CT or MRI) in addition to $\mathrm{x}$-ray to assess for the presence or absence of subchondral fracture/collapse. Uniform treatment interventions must be used across the entire treatment cohorts to qualify for inclusion. In addition, radiographic evidence of disease progression (or lack thereof) must be included as an endpoint, not just conversion to THA. Also, studies must include a minimum follow-up period of 2 years in order meet eligibility criteria (Table 1).

Interventions, Outcomes, and Comparators of Interest

For PICO \#1, we aim to compare x-ray, CT, and MRI for diagnosing and monitoring the progression of ONFH. Specifically, we will compare sensitivities and specificities of each modality for (1) diagnosing 
ONFH; (2) detecting a subchondral fracture; (3) monitoring the effect of any intervention; and (4) characterizing symptomatic versus asymptomatic disease.

For PICO \#2, various modalities for the treatment of ARCO stage I-II ONFH will be compared. These treatments may include conservative methods (e.g. physical therapy, oral medication, hyperbaric oxygen), core decompression alone, core decompression with adjunctive bone grafting (vascularized and nonvascularized autologous bone grafts, allogeneic bone, synthetic bone substitutes) or core decompression with ancillary cell-based therapy. The primary outcome of interest for PICO \#2 will be to determine which treatment modality is most effective in preventing femoral head subchondral fracture. In addition, we will aim to compare the rates of subchondral femoral head fracture in asymptomatic patients who undergo treatment versus serial observation. We will also attempt to determine if symptomatic patients who ARCO stage I-II ONFH should undergo THA to reduce hip pain.

For PICO \#3, a comparison of surgical treatment methods for patients who have ARCO stage III ONFH will be performed. These treatment modalities will include rotational osteotomy, hemiarthroplasty, surface replacement arthroplasty, THA and others. We will aim to determine which surgical treatment yields the best functional outcome over time frames of (1) less than 25 years; and (2) greater than 25 years. In doing so, we hope to elucidate which treatment methods are most appropriate for older patient populations ( $<25$-year time frame) compared to their younger counterparts ( $>25$-year time frame).

\section{Information Sources and Search Strategy}

Medline (via Ovid and PubMed), EMBASE (via Ovid), Web of Science Core Collection, SCOPUS, Global Index Medicus, and The Cochrane Library (via Wiley) were utilized as databases for our literature search.

The search strategies for identifying studies for each PICO question were created with the assistance of a research librarian (CB). They were then refined using the commentary from the remaining board members. Table 2 outlines the search strategies that were approved for each of the three research questions. Following the creation of these search strategies, participants were assigned to each of the three PICO groups and the agenda and goals for each research question were established.

Following the first literature screening performed by the members of each PICO group, a second virtual workshop was held to discuss full-text literature screening and data abstraction. After this workshop, in order to ensure the veracity and thoroughness of the literature search, all participants were asked to review the literature captured by the searches for any missing articles, review the search strategies, and submit any potential missing articles. This step was implemented in order to enhance the comprehensiveness and confidence of the literature searches. Furthermore, once this supplemental manual review was completed, a survey of all PICO group members was undertaken to ensure that consensus had been achieved with respect to the adequacy of the literature search. 
A web application (Rayyan, Qatar Computing Research Institute, https://rayyan.qcri.org) was used to facilitate effective collaboration among workshop members. The members of each PICO group independently screened the titles and abstracts of all records identified in their respective literature searches. The group chairs for PICO \#1 (EYC), PICO \#2 (QC), and PICO \#3 (SBG) resolved any discrepancies that resulted from the initial reviews.

At this point, the group chairs were confident that the best evidence to use for supporting a CPG were captured. To ensure consensus at this step in the development process, a survey of all participants was undertaken to verify that the gathered evidence and CPG development process were appropriate and acceptable. There was unanimous agreement to proceed with full-text screening, assessment of methodological quality, data abstraction, and evidence synthesis as preparation for the development of recommendations.

\section{Handling Missing Data}

In the case that any data reported in a study are unclear or not available, we will email the corresponding author to request the missing data, as well as any other unpublished data. If there is no response, an additional email will be sent 2 weeks later. If 2 weeks elapse following the second email, we will attempt to contact the first author or senior author (if not listed for correspondence). All attempts to contact these authors will be documented.

\section{Risk of Bias in Individual Studies}

Two independent reviewers from each PICO group will assess the risk of bias for each study using validated instruments to evaluate methodological quality and quantify study limitations. The instrument used will depend on the study design. For studies evaluating diagnostic accuracy (PICO \#1), the Quality Assessment of Diagnostic Accuracy Studies (QUADAS) tool $[19,20]$ will be utilized to evaluate the risk of bias. For non-randomized observational studies, the Newcastle-Ottawa Scale (NOS) [21] or the ROBINS-I scale [22] will be used for methodological quality assessment. Lastly, the risk of bias tool (RoB 2) developed by the Cochrane Collaboration will be used for risk of bias assessment for randomized control trials [23]. The risk of bias will also be assessed across multiple studies (Meta-bias) including the detection of publication bias.

\section{Data Synthesis}

Extracted data will be organized according to outcome of interest within each PICO question in order to determine if quantitative synthesis is appropriate. At least two authors will extract data from each included study and record the pertinent information in a shared spreadsheet. If any disagreement occurs in the data extracted by individual authors, the group chair will resolve the discrepancy. Heterogeneity will be assessed using the $\mathrm{I}^{2}$ statistic, and an $\mathrm{I}^{2}$ of above $40 \%$ will be considered significant. Should there be significant heterogeneity, no quantitative synthesis will be undertaken. If data is pooled, sensitivity analysis may be conducted to ensure that no one study has undue influence on a particular outcome. If 
quantitative synthesis is inappropriate, data will be presented in a tabular and narrative format and organized to address each recommendation and its associated outcomes. The quality of evidence used to synthesize each recommendation will be assessed using the GRADE (Grading of Recommendations, Assessment, Development and Evaluations) rating system.[24]

\section{Discussion}

There are many treatment options for patients who have osteonecrosis of the femoral ONFH and management strategies vary widely both among and within individual countries. Therefore, the ARCO board sought to develop a high-quality CPG. In order to do so, they consulted with the American Academy of Orthopaedic Surgeons and other outside consultants. A research librarian (CB) with expertise in systematic reviews and CPG's was retained to both lead and guide proper procedural development and adherence to the AGREE II instrument was undertaken to apply the required rigor to create a clinically useful and meaningful CPG free of bias [13]. Given the 3 PICO research questions, a participant group for each question was established composed of international experts drawn from within ARCO (Table 3). Each group was led by a chair, who also represented the group at the leadership level. An independent website (https://sites.google.com/umn.edu/arcocpg/home) was established to provide a central repository for the protocol, forms, and study files readily accessible by all participants at any time. The broad international representation spanning 16 time zones created logistical challenges for holding virtually based workshops. Nonetheless, over $95 \%$ of the participants attended these workshops and in addition, a video recording of the workshop, along with the slide deck, was posted on the project website. Due to the 2019 coronavirus pandemic, the first workshop was held virtually in March 2020 to review the CPG process and PICO questions, to train participants for literature screening, and to agree upon the inclusion and exclusion criteria that were used to determine the eligibility of identified articles.

\section{Rationale for PICO \#1:}

ONFH frequently follows a progressive course with a majority of lesions leading to joint collapse $[25,26]$. Femoral head collapse typically occurs within two years of disease onset [26,27], but could sometimes progress more slowly in the setting of smaller asymptomatic lesions [28]. The occurrence of joint collapse necessitates THA. However, when identified in its early stages, joint preserving strategies can be employed to delay or prevent the need for THA. Because this disease tends to affect younger patients, the importance of prompt diagnosis and treatment cannot be overstated. Due to the difficulty in determining the best diagnostic and prognostic signs of $\mathrm{ONFH}$, the available classification systems continue to evolve. As previously stated, ARCO recently published a newly revised iteration of their classification system [11]. Although it is hoped this system will be universally utilized, further work is needed to assess the importance of prognostic factors including lesion size, location, and acetabular involvement. In addition, it is imperative to determine the optimal imaging modalities for diagnosing ONFH, staging the extent of lesions, and monitoring the course of disease. While standard radiographs can sometimes be sufficient for the diagnosis and characterization of a given lesion, it is important to delineate when magnetic resonance (MR) or computed tomography (CT) imaging studies should be pursued. The above 
concepts and issues concerned with imaging, diagnosis, and classification of the disease need to be evaluated, and are the subject of the first research question in the development of these proposed guidelines.

\section{Rationale for PICO \#2}

Given the natural history of ONFH, it is clear that a high priority goal of treatment is to prevent subchondral collapse when lesions are diagnosed in the early stages of disease. For example, many studies support core decompression as a safe and effective treatment method that outperforms nonoperative management for pre-collapse lesions [25, 27, 29, 30]. Nevertheless, some surgeons advocate core decompression only for smaller lesions, as the results are not as favorable for larger lesions [31]. However, some smaller lesions have been shown to have a better prognosis regardless of any intervention, and at times, may even resolve spontaneously [32, 33]. This raises the question of when core decompression should be performed for a clear benefit, and recent meta-analyses and clinical trials have questioned its efficacy $[30,34,35]$. This has led to surgeons modifying either the technical procedure or augmenting the procedure biologically with grafts, growth factors, cell based therapies, or synthetic bone substitutes. While small-diameter drilling may provide the same clinical benefit as traditional core decompression [36,37], the implications of performing one variant versus the other is not fully understood. In addition, attempts have been made to enhance the results of core decompression with various cell-based adjuncts, bone graft preparations, and bone morphogenetic proteins. When considering stand-alone bone grafting procedures, there is an assortment of techniques (e.g., vascularized and non-vascularized grafts) and harvest sites, as well as varying approaches for graft insertion [38-51]. Various other procedures including multiple types of osteotomies can also be utilized in an attempt to preserve the femoral head [52-58]. Without evidence-based CPGs, it is difficult to establish which strategy is ideal across various patient populations. Thus, these strategies are the subject of the second research question in the development of these proposed guidelines.

\section{Rationale for PICO \#3}

For patients whose femoral head is no longer spherical due to impaction of a segment of underlying subchondral bone (collapse), THA is typically the treatment of choice for most patients. The clinical challenge is that all too often, osteonecrosis develops in a teenager or young adult. Despite the excellent pain relief afforded by hip arthroplasty, the long-term performance and durability of a current hip arthroplasty implanted in patients who have a life expectancy of over 25 years is unknown. For these patients, attempts to preserve the femoral head by performing osteotomies or bone grafting procedures might delay or prevent the need for an arthroplasty, but the degree of improvement in pain and function may be suboptimal. In contrast to traditional THA implants, short-stem femoral components are sometimes used in an attempt to preserve metaphyseal bone stock [59-64]. However, the true implications of employing these implant designs in the setting of osteonecrosis has not been confirmed. In addition, other treatment strategies including, hemiarthroplasty, and surface replacement arthroplasty may be viable options. However, the surgical treatment that yields the best pain relief and functional outcome has 
not been clearly established. To this end, the ARCO has set out to establish evidence-based CPG for the treatment of ONFH.

In summary, the project methodology has progressed as follows:

- 09/2019: ARCO board votes to pursue CPG at Orlando 2020 workshop

- 10/2019: Exploratory discussions with AAOS undertaken

- 10/2019: Research services liaison joins CPG process as content expert and facilitator

- 12/2019: PICO questions proposed, circulated to board for comment and approval

- 11/ 2019 - 01/2020: Orlando workshop agenda for development of CPG established

- 01/2020 - 02/ 2020: Literature search strategy was refined in preparation for workshop; web-based platform (Rayyan) tested for usage by workshop participants

- 02/2020: Workshop participants assigned to PICO groups and agenda/goals established

- 03/2020: First workshop on CPG held virtually due to COVID-19 pandemic. PICO questions and CPG process reviewed, training given for literature screening, inclusion and exclusion criteria agreed upon by participants

- 04/2020: First literature screening completed

- 05/2020: Second workshop held virtually, full-text screening and data abstraction training.

- 05/2020 - 06/2020: Open period to submit potential missing articles and review/revise search strategy

- 06/2020: Conference of PICO group chairs and CPG expert

- 07/2020: Full text screening

- 08/2020: Assess Methodological Quality

- 09/2020 - 11/2020: Synthesize Evidence

- 12/2020 - 2/2021: Develop Recommendations

\section{Summary}

Guidelines for the optimal method of diagnosing ONFH and identifying subchondral fracture or femoral head collapse have not been clearly established. For patients who have ONFH without subchondral fracture, the best treatment method for preventing progression of disease is yet to be clearly elucidated. In addition, for patients who have ARCO stage 3 lesions with femoral head subchondral fracture, the surgical treatment that yields the best clinical outcomes is not fully understood. By pursuing the development of evidence-based CPGs for each of these three research questions, the ARCO board hopes to provide clinicians and patients guidance in determining the most appropriate diagnostic and therapeutic strategies ONFH.

\section{List Of Abbreviations}


ONFH- Osteonecrosis of the femoral head

ARCO- Association Research Circulation Osseous

CPG- Clinical practice guidelines

PRISMA- Preferred reporting Items for Systematic Reviews and Meta-Analyses

PICO- Patient, Intervention, Comparison, and Outcome

CT- Computed tomography

MRI- Magnetic resonance imaging

THA- Total hip arthroplasty

QUADAS- Quality Assessment of Diagnostic Accuracy Studies

NOS-Newcastle-Ottawa Scale

AAOS- American Academy of Orthopaedic Surgeons

\section{Declarations}

\section{Ethics approval and consent to participate}

Not applicable

Consent for publication

Not applicable

\section{Availability of data and materials}

The datasets used and/or analyzed during the current study are available from the corresponding author on reasonable request.

\section{Competing interests}

EYC: Association Research Circulation Osseous International (ARCO): Board or committee member Innomed: IP royalties

Journal of Bone and Joint Surgery - American: Editorial or governing board; Publishing royalties, financial or material support

Musculoskeletal Transplant Foundation: Board or committee member; Other financial or material support 
Rein in Sarcoma (Research grant): Research support

Smith \& Nephew: Other financial or material support

MAM:

AAOS: Board or committee member

American Association of Hip and Knee Surgeons: Board or committee member

CERAS Health: Stock or stock Options

Cymedica: Paid consultant

Flexion Therapeutics: Paid consultant

Johnson \& Johnson: Paid consultant; Research support

Journal of Arthroplasty: Editorial or governing board

Journal of Knee Surgery: Editorial or governing board

Knee Society: Board or committee member

Kolon TissueGene: Paid consultant

Medicus Works LLC: Publishing royalties, financial or material support

MirrorAR: Stock or stock Options

National Institutes of Health (NIAMS \& NICHD): Research support

Orthopedics: Editorial or governing board

Pacira: Paid consultant

Peerwell: Stock or stock Options

Pfizer: Paid consultant

RegenLab: Research support

Stryker: IP royalties; Paid consultant; Research support

Surgical Techniques International: Editorial or governing board

TissueGene: Research support 
Up-to Date: Publishing royalties, financial or material support

USMI: Stock or stock Options

Wolters Kluwer Health - Lippincott Williams \& Wilkins: Publishing royalties, financial or material support

SBG:

Accelalox: Stock or stock Options; Unpaid consultant

ARCO: Board or committee member

Arquos: Stock or stock Options

Bioengineering: Editorial or governing board

Biomaterials: Editorial or governing board; Publishing royalties, financial or material support

Bone and Joint Research: Editorial or governing board

Clinical Orthopaedics and Related Research: Editorial or governing board

Hyalex: IP royalties; Stock or stock Options

J Arthroplasty: Editorial or governing board

J Biomed Mater Res: Editorial or governing board

Journal of Orthopaedic Research: Editorial or governing board; Publishing royalties, financial or material support

Journal of Orthopaedic Translation: Editorial or governing board

Merck Manual: Publishing royalties, financial or material support

Open Orthopaedics Journal: Editorial or governing board

Orthopedics: Editorial or governing board

PLOS ONE: Editorial or governing board

Pluristem: Paid consultant

Regenerative Engineering and Translational Medicine: Editorial or governing board

Wishbone Medical: Paid consultant

RJS: 
American Association of Hip and Knee Surgeons: Board or committee member

Anchor study group: Board or committee member

Biomet: Paid consultant; Paid presenter or speaker

Cytori: Research support

DePuy, A Johnson \& Johnson Company: Research support

Journal of Arthroplasty: Editorial or governing board

Knee Society: Board or committee member

Link Orthopaedics: IP royalties; Paid consultant

Midamerica orthopedic society: Board or committee member

Muller Foundation: Board or committee member

Orthalign: IP royalties

Orthoalign: Paid consultant; Stock or stock Options

Springer: Publishing royalties, financial or material support

Stryker, Biomet: Research support

Think: Paid consultant

Zimmer: IP royalties; Research support

QC:

ARCO: Board or committee member

DePuy, A Johnson \& Johnson Company: Research support

Exactech, Inc: Paid consultant; Research support

Journal of Arthroplasty: Editorial or governing board

Journal of Orthopaedic Research: Editorial or governing board

Saunders/Mosby-Elsevier: Publishing royalties, financial or material support

Virginia Orthopaedic Society: Board or committee member 
HSS:

No competing interests to disclose

\section{Funding:}

This study was not funded.

\section{Authors' contributions}

The listed authors have drafted and edited the manuscript. All authors have approved the submitted version of the manuscript and have agreed both to be personally accountable for the work, ensure that questions related to the accuracy or integrity of any part of the work, even ones in which the author was not personally involved, are appropriately investigated, resolved, and documented in the literature. The entire Clinical Practice Guideline group has reviewed, submitted edits, and approved the protocol.

\section{Acknowledgements}

ARCO Clinical Practice Guideline workgroup, in addition to listed authors (in alphabetical order):

Ando, Wataru ; Baek, Seung-Hoon; Bakker, Caitlin J; Drescher, Wolf; Ha, Yong-Chan; Hernigou, Philippe; Jones, Lynne C; Kim, Harry; Kim, Shin-Yoon; Kim, Tae-Young; Koo, Kyung-Hoi; Lee, Mel; Reichert, Ines; Sakai, Takashi; Stronach, Ben; Sugano, Nobuo; Yamamoto, Takuaki; Yoon, Byung-Ho; and Zhao, Dewei Institutional affiliations for ARCO CPG workgroup: 
Wataru Ando, MD, -ando@umin.ac.jp

$\mathrm{PhD}$

Caitlin Bakker, MLIS cjbakker@umn.edu

Seung-Hoon Baek, sbaek@knu.ac.kr MD PhD

Edward Y. Cheng, cheng002@umn.edu MD

Quanjun Cui, MD

Wolf Drescher, MD

Stuart Goodman, $\mathrm{MD}, \mathrm{PhD}$

Yong-Chan $\mathrm{Ha}$, MD

Philippe Hernigou, MD

Lynne C. Jones, PhDｌjones3@jhmi.edu

harry.kim@tsrh.org

Harry K. W. Kim, MD

Shin-Yoon Kim, MD,

$\mathrm{PhD}$

Tae-Young Kim, MD, syty-chan@hanmail.net $\mathrm{PhD}$

Kyung-Hoi Koo, MDｋhkoo@snu.ac.kr

Mel Lee, MD, PhDｂone@doctor.com

Michael Mont, MD rhondamont@aol.com

Ines Reichert, MD, ines.reichert@kcl.ac.uk

$\mathrm{PhD}$

Takashi Sakai, MD,

PhD

Rafael J. Sierra, MD

Benjamin M

Stronach, MD

Nobuhiko Sugano,

$\mathrm{MD}, \mathrm{PhD}$

cozy@yamaguchi-u.ac.jp

sierra.rafael@mayo.edu

bstronach@umc.edu

n-sugano@umin.net

Takuaki Yamamoto, $\mathrm{MD}, \mathrm{PhD}$
Osaka University, Osaka, Japan

University of Minnesota, Minneapolis, USA

Kyungpook National University, Daegu, South Korea

University of Minnesota, Minneapolis, USA

University of Virginia, Charlottesville, USA

Department of Orthopedics, RWTH

Aachen University, Aachen, Germany

Stanford University, Palo Alto, USA

Chung-Ang University, Seoul, South Korea

Hôpital Henri Mondo, Paris, France

Johns Hopkins University, Baltimore, USA

Scottish Rite for Children, Dallas, USA

Kyungpook National University, Daegu, South Korea

Konkuk University, Seoul, South Korea

Seoul National University College of Medicine, Seoul, South Korea

Chang Gung Memorial Hospital, Kaohsiung, Taiwan

Lenox Hill Hospital, New York, USA

Kings College Hospital, London, United Kingdom

Yamaguchi University Graduate School of Medicine, Ube, Japan

Mayo Clinic, Rochester, USA

University of Mississippi, Jackson, USA

Osaka University, Osaka, Japan

Fukuoka University, Fukuoka City, Japan 
Byung-Ho Yoon, MD, cragy0215@naver.com Inje University, Seoul, South Korea $\mathrm{PhD}$

Dewei Zhao, MD

zhaodewei2000@163.com

Zhongshan Hospital of Dalian University, Dalian, China

\section{References}

1. Moya-Angeler J, Gianakos AL, Villa JC, Ni A, Lane JM. Current concepts on osteonecrosis of the femoral head. World J Orthop. 2015;6(8):590-601.

2. Ikeuchi K, Hasegawa Y, Seki T, Takegami Y, Amano T, Ishiguro N. Epidemiology of nontraumatic osteonecrosis of the femoral head in Japan. Modern rheumatology. 2015;25(2):278-81.

3. Kang JS, Moon KH, Kwon DG, Shin BK, Woo MS. The natural history of asymptomatic osteonecrosis of the femoral head. Int Orthop. 2013;37(3):379-84.

4. Zhao DW, Yu M, Hu K, Wang W, Yang L, Wang BJ, et al. Prevalence of Nontraumatic Osteonecrosis of the Femoral Head and its Associated Risk Factors in the Chinese Population: Results from a Nationally Representative Survey. Chin Med J (Engl). 2015;128(21):2843-50.

5. Mankin HJ. Nontraumatic necrosis of bone (osteonecrosis). N Engl J Med. 1992;326(22):1473-9.

6. Lespasio MJ, Sodhi N, Mont MA. Osteonecrosis of the Hip: A Primer. Perm J. 2019;23.

7. Mont MA, Hungerford DS. Non-traumatic avascular necrosis of the femoral head. J Bone Joint Surg Am. 1995;77(3):459-74.

8. Mont MA, Salem HS, Piuzzi NS, Goodman SB, Jones LC. Nontraumatic Osteonecrosis of the Femoral Head: Where Do We Stand Today?: A 5-Year Update. J Bone Joint Surg Am. 2020.

9. Yoon BH, Jones LC, Chen $\mathrm{CH}$, Cheng EY, Cui Q, Drescher W, et al. Etiologic Classification Criteria of ARCO on Femoral Head Osteonecrosis Part 2: Alcohol-Associated Osteonecrosis. J Arthroplasty. 2019;34(1):169-74.e1.

10. Yoon BH, Jones LC, Chen CH, Cheng EY, Cui Q, Drescher W, et al. Etiologic Classification Criteria of ARCO on Femoral Head Osteonecrosis Part 1: Glucocorticoid-Associated Osteonecrosis. J Arthroplasty. 2019;34(1):163-8.e1.

11. Yoon B-H, Mont MA, Koo K-H, Chen C-H, Cheng EY, Cui Q, et al. The 2019 revised version of Association Research Circulation Osseous staging system of osteonecrosis of the femoral head. The Journal of Arthroplasty.

12. Institute of Medicine Committee on Standards for Developing Trustworthy Clinical Practice G. In: Graham R, Mancher M, Miller Wolman D, Greenfield S, Steinberg E, editors. Clinical Practice Guidelines We Can Trust. Washington (DC): National Academies Press (US) Copyright 2011 by the National Academy of Sciences. All rights reserved.; 2011.

13. Brouwers MC, Kho ME, Browman GP, Burgers JS, Cluzeau F, Feder G, et al. AGREE II: advancing guideline development, reporting and evaluation in health care. Cmaj. 2010;182(18):E839-42. 
14. Moher D, Liberati A, Tetzlaff J, Altman DG. Preferred reporting items for systematic reviews and meta-analyses: the PRISMA statement. PLoS Med. 2009;6(7):e1000097.

15. Atkins D, Best D, Briss PA, Eccles M, Falck-Ytter Y, Flottorp S, et al. Grading quality of evidence and strength of recommendations. Bmj. 2004;328(7454):1490.

16. Brozek JL, Akl EA, Alonso-Coello P, Lang D, Jaeschke R, Williams JW, et al. Grading quality of evidence and strength of recommendations in clinical practice guidelines. Part 1 of 3 . An overview of the GRADE approach and grading quality of evidence about interventions. Allergy. 2009;64(5):66977.

17. Guyatt GH, Oxman AD, Vist GE, Kunz R, Falck-Ytter Y, Alonso-Coello P, et al. GRADE: an emerging consensus on rating quality of evidence and strength of recommendations. Bmj. 2008;336(7650):924-6.

18. Qaseem A, Forland F, Macbeth F, Ollenschläger G, Phillips S, van der Wees P. Guidelines International Network: toward international standards for clinical practice guidelines. Ann Intern Med. 2012;156(7):525-31.

19. Whiting P, Rutjes AW, Reitsma JB, Bossuyt PM, Kleijnen J. The development of QUADAS: a tool for the quality assessment of studies of diagnostic accuracy included in systematic reviews. BMC Med Res Methodol. 2003;3:25.

20. Whiting PF, Rutjes AW, Westwood ME, Mallett S, Deeks JJ, Reitsma JB, et al. QUADAS-2: a revised tool for the quality assessment of diagnostic accuracy studies. Ann Intern Med. 2011;155(8):529-36.

21. Higgins JPT. Cochrane Handbook for Systematic Reviews of Interventions Version 5.0.1. The Cochrane Collaboration. http://wwwcochrane-handbookorg. 2008.

22. Sterne JA, Hernán MA, Reeves BC, Savović J, Berkman ND, Viswanathan M, et al. ROBINS-l: a tool for assessing risk of bias in non-randomised studies of interventions. Bmj. 2016;355:i4919.

23. Sterne JAC, Savović J, Page MJ, Elbers RG, Blencowe NS, Boutron I, et al. RoB 2: a revised tool for assessing risk of bias in randomised trials. Bmj. 2019;366:14898.

24. Guyatt G, Oxman AD, Sultan S, Brozek J, Glasziou P, Alonso-Coello P, et al. GRADE guidelines: 11. Making an overall rating of confidence in effect estimates for a single outcome and for all outcomes. J Clin Epidemiol. 2013;66(2):151-7.

25. Mont MA, Carbone JJ, Fairbank AC. Core decompression versus nonoperative management for osteonecrosis of the hip. Clin Orthop Relat Res. 1996(324):169-78.

26. Mont MA, Zywiel MG, Marker DR, McGrath MS, Delanois RE. The natural history of untreated asymptomatic osteonecrosis of the femoral head: a systematic literature review. J Bone Joint Surg Am. 2010;92(12):2165-70.

27. Roth A, Beckmann J, Bohndorf K, Fischer A, Heiss C, Kenn W, et al. S3-Guideline non-traumatic adult femoral head necrosis. Arch Orthop Trauma Surg. 2016;136(2):165-74.

28. Hernigou P, Poignard A, Nogier A, Manicom O. Fate of very small asymptomatic stage-I osteonecrotic lesions of the hip. J Bone Joint Surg Am. 2004;86(12):2589-93. 
29. Stulberg BN, Davis AW, Bauer TW, Levine M, Easley K. Osteonecrosis of the femoral head. A prospective randomized treatment protocol. Clin Orthop Relat Res. 1991(268):140-51.

30. Hua KC, Yang XG, Feng JT, Wang F, Yang L, Zhang H, et al. The efficacy and safety of core decompression for the treatment of femoral head necrosis: a systematic review and meta-analysis. $J$ Orthop Surg Res. 2019;14(1):306.

31. Yoon TR, Song EK, Rowe SM, Park CH. Failure after core decompression in osteonecrosis of the femoral head. Int Orthop. 2001;24(6):316-8.

32. Cheng EY, Thongtrangan I, Laorr A, Saleh KJ. Spontaneous resolution of osteonecrosis of the femoral head. J Bone Joint Surg Am. 2004;86(12):2594-9.

33. Kopecky KK, Braunstein EM, Brandt KD, Filo RS, Leapman SB, Capello WN, et al. Apparent avascular necrosis of the hip: appearance and spontaneous resolution of MR findings in renal allograft recipients. Radiology. 1991;179(2):523-7.

34. Koo KH, Kim R, Ko GH, Song HR, Jeong ST, Cho SH. Preventing collapse in early osteonecrosis of the femoral head. A randomised clinical trial of core decompression. J Bone Joint Surg Br. 1995;77(6):870-4.

35. Yoon BH, Lee YK, Kim KC, Ha YC, Koo KH. No differences in the efficacy among various core decompression modalities and non-operative treatment: a network meta-analysis. Int Orthop. 2018;42(12):2737-43.

36. Brown PJ, Mannava S, Seyler TM, Plate JF, Van Sikes C, Stitzel JD, et al. Multiple Small Diameter Drillings Increase Femoral Neck Stability Compared with Single Large Diameter Femoral Head Core Decompression Technique for Avascular Necrosis of the Femoral Head. Surg Technol Int. 2016;29:247-54.

37. Cilla M, Checa S, Preininger B, Winkler T, Perka C, Duda GN, et al. Femoral head necrosis: A finite element analysis of common and novel surgical techniques. Clin Biomech (Bristol, Avon). 2017;48:49-56.

38. Chen CC, Lin CL, Chen WC, Shih HN, Ueng SW, Lee MS. Vascularized iliac bone-grafting for osteonecrosis with segmental collapse of the femoral head. J Bone Joint Surg Am. 2009;91(10):2390-4.

39. Chen X, Tan X, Gao S, Zhang X, Li J, Liu Y. Sartorius muscle-pedicle bone graft for osteonecrosis of the femoral head. Int Orthop. 2016;40(7):1417-25.

40. Feng W, Chen J, Wu K, Lu L, Deng P, Ye P, et al. A comparative study of cortico-cancellous iliac bone graft with or without the combination of vascularized greater trochanter flap for the management of femoral head osteonecrosis: a minimum 6 years follow-up. BMC musculoskeletal disorders. 2019;20(1):298.

41. Xie H, Wang B, Tian S, Liu B, Qin K, Zhao D. Retrospective Long-Term Follow-Up Survival Analysis of the Management of Osteonecrosis of the Femoral Head With Pedicled Vascularized Iliac Bone Graft Transfer. J Arthroplasty. 2019;34(8):1585-92. 
42. Yang F, Wei Q, Chen X, Hong G, Chen Z, Chen Y, et al. Vascularized pedicle iliac bone grafts as a hippreserving surgery for femur head necrosis: a systematic review. J Orthop Surg Res. 2019;14(1):270.

43. Zeng YR, He S, Feng WJ, Li FL, Li J, Jian LY, et al. Vascularised greater trochanter bone graft, combined free iliac flap and impaction bone grafting for osteonecrosis of the femoral head. Int Orthop. 2013;37(3):391-8.

44. Zhao D, Xie H, Xu Y, Wang Y, Yu A, Liu Y, et al. Management of osteonecrosis of the femoral head with pedicled iliac bone flap transfer: A multicenter study of 2190 patients. Microsurgery. 2017;37(8):896-901.

45. Cao L, Guo C, Chen J, Chen Z, Yan Z. Free Vascularized Fibular Grafting Improves Vascularity Compared With Core Decompression in Femoral Head Osteonecrosis: A Randomized Clinical Trial. Clin Orthop Relat Res. 2017;475(9):2230-40.

46. Kim SY, Kim YG, Kim PT, Ihn JC, Cho BC, Koo KH. Vascularized compared with nonvascularized fibular grafts for large osteonecrotic lesions of the femoral head. J Bone Joint Surg Am. 2005;87(9):2012-8.

47. Lakshminarayana S, Dhammi IK, Jain AK, Bhayana H, Kumar S, Anshuman R. Outcomes of Core Decompression with or without Nonvascularized Fibular Grafting in Avascular Necrosis of Femoral Head: Short Term Followup study. Indian J Orthop. 2019;53(3):420-5.

48. Plakseychuk A. CORR Insights((R)): Free Vascularized Fibular Grafting Improves Vascularity Compared With Core Decompression in Femoral Head Osteonecrosis: A Randomized Clinical Trial. Clin Orthop Relat Res. 2017;475(9):2241-4.

49. Plakseychuk AY, Kim SY, Park BC, Varitimidis SE, Rubash HE, Sotereanos DG. Vascularized compared with nonvascularized fibular grafting for the treatment of osteonecrosis of the femoral head. J Bone Joint Surg Am. 2003;85(4):589-96.

50. Unal MB, Cansu E, Parmaksizoglu F, Cift H, Gurcan S. Treatment of osteonecrosis of the femoral head with free vascularized fibular grafting: Results of 7.6-year follow-up. Acta Orthop Traumatol Turc. 2016;50(5):501-6.

51. Meyers MH, Jones RE, Bucholz RW, Wenger DR. Fresh autogenous grafts and osteochondral allografts for the treatment of segmental collapse in osteonecrosis of the hip. Clin Orthop Relat Res. 1983(174):107-12.

52. Kawano K, Motomura G, Ikemura S, Kubo Y, Fukushi J, Hamai S, et al. Long-term hip survival and factors influencing patient-reported outcomes after transtrochanteric anterior rotational osteotomy for osteonecrosis of the femoral head: A minimum 10-year follow-up case series. Modern rheumatology. 2018:1-7.

53. Kubo Y, Motomura G, Ikemura S, Hatanaka H, Utsunomiya T, Hamai S, et al. Effects of anterior boundary of the necrotic lesion on the progressive collapse after varus osteotomy for osteonecrosis of the femoral head. J Orthop Sci. 2019.

54. Lakhotia D, Swaminathan S, Shon WY, Oh JK, Moon JG, Dwivedi C, et al. Healing Process of Osteonecrotic Lesions of the Femoral Head Following Transtrochanteric Rotational Osteotomy: A 
Computed Tomography-Based Study. Clin Orthop Surg. 2017;9(1):29-36.

55. Lee YK, Park CH, Ha YC, Kim DY, Lyu SH, Koo KH. Comparison of Surgical Parameters and Results between Curved Varus Osteotomy and Rotational Osteotomy for Osteonecrosis of the Femoral Head. Clin Orthop Surg. 2017;9(2):160-8.

56. Mont MA, Fairbank AC, Krackow KA, Hungerford DS. Corrective osteotomy for osteonecrosis of the femoral head. J Bone Joint Surg Am. 1996;78(7):1032-8.

57. Sugioka Y, Hotokebuchi T, Tsutsui H. Transtrochanteric anterior rotational osteotomy for idiopathic and steroid-induced necrosis of the femoral head. Indications and long-term results. Clin Orthop Relat Res. 1992(277):111-20.

58. Sugioka Y, Yamamoto T. Transtrochanteric posterior rotational osteotomy for osteonecrosis. Clin Orthop Relat Res. 2008;466(5):1104-9.

59. Suksathien Y, Sueajui J. Mid-term results of short stem total hip arthroplasty in patients with osteonecrosis of the femoral head. Hip international : the journal of clinical and experimental research on hip pathology and therapy. 2018:1120700018816011.

60. Schnurr C, Loucif A, Patzer T, Schellen B, Beckmann J, Eysel P. Short stem survival after osteonecrosis of the femoral head. Arch Orthop Trauma Surg. 2018;138(4):573-9.

61. Kim YH, Park JW. Ultra-Short Anatomic Uncemented Femoral Stem and Ceramic-on-Ceramic Bearing in Patients With Idiopathic or Ethanol-Induced Femoral Head Osteonecrosis. J Arthroplasty. 2019.

62. Gallart X, Fernandez-Valencia JA, Rios G, Bori G, Riba J, Munoz-Mahamud E, et al. Early clinical and radiological outcomes for the Taperloc Complete Microplasty stem. Eur J Orthop Surg Traumatol. 2019;29(3):619-24.

63. Capone A, Bienati F, Torchia S, Podda D, Marongiu G. Short stem total hip arthroplasty for osteonecrosis of the femoral head in patients 60 years or younger: a 3- to 10-year follow-up study. BMC musculoskeletal disorders. 2017;18(1):301.

64. Miladi M, Villain B, Mebtouche N, Begue T, Auregan JC. Interest of short implants in hip arthroplasty for osteonecrosis of the femoral head: comparative study "uncemented short" vs "cemented conventional" femoral stems. Int Orthop. 2018;42(7):1669-74.

\section{Tables}

Table 1. Inclusion and Exclusion Criteria for each Research Question 
Inclusion:

Focus of article is on imaging, not on a treatment outcome

Humans only

Adult population only (age $\geq 16$ years)

Exclusion

Animal studies

Foreign language without full text English translation

Single case reports

articles that do not present original data (e.g., reviews, editorials)
Inclusion:

Evidence level 1-4

- $\quad$ Case series $>15$ patients

. Human only

- Prospective or retrospective

- $\quad$ Diagnosis $=$ non-traumatic $\mathrm{ONFH}$

. $\quad$ Age $\geq 16$ year

. $\quad$ Advanced imaging (CT or MRI) in addition to $\mathrm{XR}$, used to assess for subchondral collapse entire treatment cohort

. Outcome includes radiographic progression not only conversion to total hip arthroplasty

. Follow-up: $\geq 2$ years

Exclusion:

Animal studies
. $\quad$ Foreign language without full text English
translation
Single case reports
No duplicate papers or patient cohorts
Articles that do not present original data (e.g.,
reviews, editorials)

Inclusion:

Evidence level 1-4
Case series $>15$ patients
Human only
Prospective or retrospective
Diagnosis $=$ non-traumatic ONFH
Age $\geq 16$ years
Staging data provided
Treatment intervention is uniform
across entire treatment cohort
Outcome data provided
Follow-up: $\geq 2$ years

Exclusion:

. Animal studies

- $\quad$ Foreign language without full text English translation
- Single case reports
- No duplicate papers or patient cohorts

- Articles that do not present original data (e.g., reviews, editorials)

Table 2. Search Strategies in Medline Format* 


\begin{tabular}{|c|c|c|}
\hline PICO \#1 & PICO \#2 & PICO \#3 \\
\hline 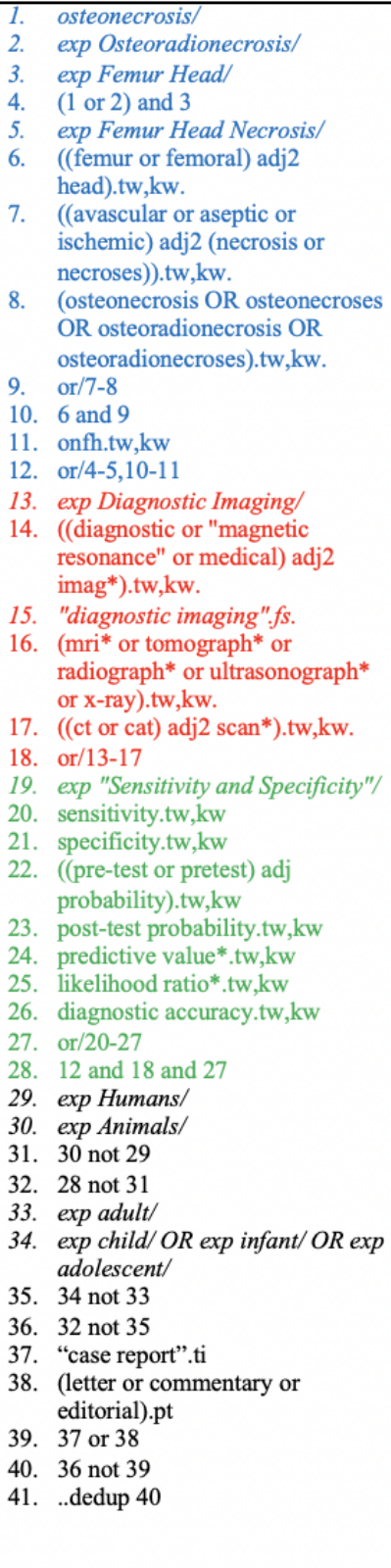 & $\begin{array}{ll}\text { 1. } & \text { osteonecrosis/ } \\
\text { 2. } & \text { exp Osteoradionecrosis/ } \\
\text { 3. } & \text { exp Femur Head/ } \\
\text { 4. } & (1 \text { or } 2 \text { ) and } 3 \\
\text { 5. } & \text { exp Femur Head Necrosis/ } \\
\text { 6. ((femur or femoral) adj2 head).tw,kw. } \\
\text { 7. ((avascular or aseptic or ischemic) adj2 } \\
\text { (necrosis or necroses)).tw,kw. } \\
\text { 8. (osteonecrosis OR osteonecroses OR } \\
\text { osteoradionecrosis OR } \\
\text { osteoradionecroses).tw,kw. } \\
\text { 9. or/7-8 } \\
\text { 10. } 6 \text { and } 9 \\
\text { 11. onfh.tw,kw } \\
\text { 12. or/4-5,10-11 } \\
\text { 13. ((fracture* or collapse*) adj2 (femur or } \\
\text { femoral) adj2 head*).tw,kw. } \\
\text { 14. exp Femur Head/ } \\
\text { 15. exp Hip Fractures/ } \\
\text { 16. } 14 \text { and } 15 \\
\text { 17. } 13 \text { or } 16 \\
\text { 18. without.mp. } \\
\text { 19. } 17 \text { and } 18 \\
\text { 20. (ARCO adj5 ("1" or "2" or One or } \\
\text { Two)).tw,kw. } \\
\text { 21. ("pre-collapse" or precollapse or (before } \\
\text { adj2 collaps*)).tw,kw. } \\
\text { 22. or/19-21 } \\
\text { 23. } 12 \text { and } 22 \\
\text { 24. exp Humans/ } \\
\text { 25. exp Animals/ } \\
\text { 26. } 25 \text { not } 24 \\
\text { 27. } 23 \text { not } 26 \\
\text { 28. exp Adult/ } \\
\text { 29. exp Child/ or exp Infant/ or exp } \\
\text { Adolescent/ } \\
\text { 30. } 29 \text { not } 28 \\
\text { 31. } 27 \text { not } 30 \\
\text { 32. "case report".ti } \\
\text { 33. (editorial OR letter OR commentary OR } \\
\text { note).pt } \\
\text { 35. } 32 \text { or } 33 \\
\text { 36. } \text {..dedup } 35\end{array}$ & 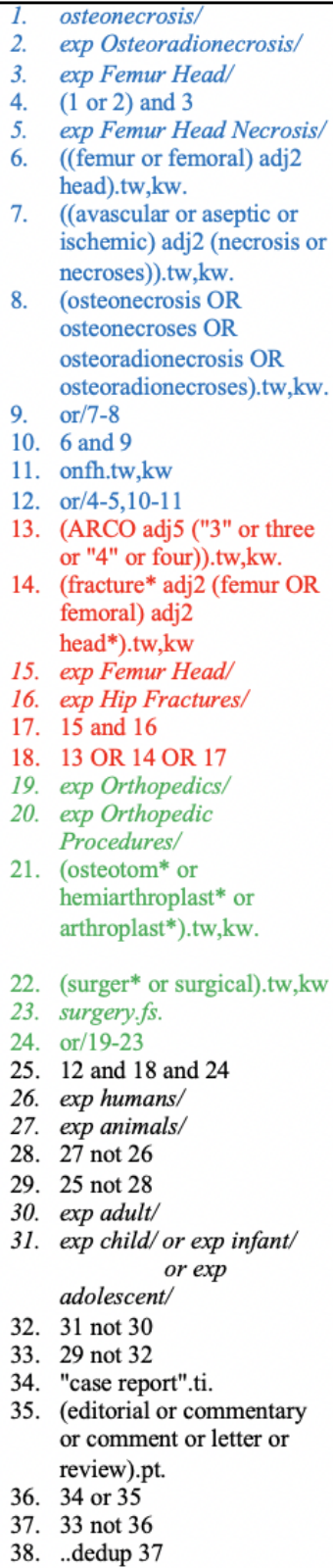 \\
\hline
\end{tabular}

*Italics refer to controlled vocabulary/taxonomy terms

Color-coding represents different concepts being captured in each search

Table 3. Group chairs and participants for each research question 


\begin{tabular}{|llll|}
\hline & PICO Group 1 & PICO Group 2 & PICO Group 3 \\
\hline Group Chair: & Edward Y. Cheng, MD & Quanjun Cui, MD & Stuart B. Goodman, MD, PhD \\
\hline Kyung-Hoi Koo, MD & Harry Kim, MD & Benjamin M. Stronach, MD \\
\hline Troup Members: & Lynne C. Jones, MD & Nobuhiko Sugano, MD & Rafael J. Sierra, MD \\
\hline Takuaki Yamamoto, MD & Phillipe Hernigou, MD & Seung-Hoon Baek, MD, PhD \\
\hline & Wolf Drescher, MD & Tae-Young Kim, MD \\
\hline & Shin-Yoon Kim, MD & Wataru Ando, MD, PhD \\
\hline
\end{tabular}

\section{Figures}




\section{PICO 1 group}

Edward Cheng MD (group chair)

Kyung-Hoi Koo, MD

Takashi Sakai

Lynne Jones, $\mathrm{PhD}$

Takuaki Yamamoto

\section{PICO 2 group}

Quanjun Cui, MD (group chair)

Harry Kim, MD

Michael Mont, MD

Nobuo Sugano MD, $\mathrm{PhD}$

Philippe Hemigou, MD

Wolf Drescher, MD

Shin-Yoon Kim, MD

Zhao Dewei, MD

Ines Reichert, $\mathrm{MD}, \mathrm{PhD}$

\section{PICO 3 group}

Stuart Goodman (group chair)

Ben Stronach

Byung-Ho Yoon

Rafael Sierra

Seung-Hoon Baek

Tae-Young Kim

Wataru Ando

Yong-Chan $\mathrm{Ha}, \mathrm{MD}$

Mel Lee, MD, PhD
University of Minnesota

Seoul National University

Yamaguchi University

Johns Hopkins University

yamamotot@fukuoka-u.ac.jp

University of Virginia

Texas Scottish Rite Hospital

Northwell Health

Osaka University

University of Paris - Est

RWTH Aachen University

Kyungpook National University

Dalian University

King's College Hospital

Stanford University

University of Mississippi

Seoul National University

Mayo Clinic

Kyungpook National University

Konkuk University

Osaka University

Chung-Ang University

Chang Gung Memorial Hospital

\section{Figure 1}

PICO study groups

\section{Supplementary Files}

This is a list of supplementary files associated with this preprint. Click to download.

- ONFHCPGPRISMAchecklist.docx 